\title{
Robust Control of Delay Systems: A Sliding Mode Control Design via LMI.
}

\author{
F. Gouaisbaut, M. Dambrine, J.P. Richard \\ LAIL (CNRS UPRESA 8021), \\ Ecole Centrale de Lille, BP 48 , \\ 59651 Villeneuve d'Ascq CEDEX - FRANCE. \\ e-mail : \{frederic.gouaisbaut, michel.dambrine, jprichard\}@ec-lille.fr
}

\begin{abstract}
This paper considers the sliding mode control of uncertain systems with single or multiple, constant or time-varying state-delays, submitted to additive perturbations. The sliding surface is designed so to maximize the calculable set of admissible delays. The conditions for the existence of the sliding regime are studied by using LyapunovKrasovskii functionals and Lyapunov-Razumikhin functions. LMIs are used for the optimization procedure. Two examples illustrate the proposed method.
\end{abstract}

Key words: Time-Delay system, Robust Control, Sliding Mode Control, LMI

\section{Introduction}

Time delays commonly occur in many dynamical systems and are a source of instability and poor performances even in a linear model [28]. For the last decade, several works have proposed criteria for the robust stabilization of time delay systems: for linear models with parameter uncertainties, see $[4,13,18,21,25,26]$ (Lyapunov-Krasovskii approach leading to LMIs) and [10] (comparison approach). The resulting control laws are of continuous, often memoryless, feedback type (see $[11,30]$ for surveys). The results concerning robustness with respect to external disturbances rely on either $H_{\infty}$ design (see $[20,26]$ and references therein), also leading to Riccati equations and LMIs, or structural approaches, such as disturbance decoupling using models over rings (see $[7,8,29]$ and references therein).

The sliding mode control approach [35], based on the use of discontinuous control laws (relays), is known to be an efficient alternative way to tackle many 
challenging problems of robust stabilization. For instance, an appropriate sliding mode strategy can achieve stabilization by "dominating" nonlinear terms and additive disturbances, provided some appropriate "matching conditions" hold (roughly speaking, the disturbances must belong to the space spanned by the input function). However, the combination of delay phenomenon with relay actuators makes the situation much more complex (see for instance [12] and the survey paper [31]) : designing a sliding controller without taking delays into account may lead to unstable or chaotic behaviors or, at least, results in highly chattering behaviors $[9,12]$. Even if the general framework of differential inclusions has been proposed in this case [17], the concrete control results are not so numerous $[1,2,6,9,14,15,19,23,33,34]$. Some others, that are relying on the Razumikhin's approach for the reaching phase analysis, are even needing complementary proofs [32] : the finite-time convergence of the sliding manifold is not ensured by this way and such combination with Filipov's theory has not been deeply studied. In [3], the considered systems have output delays and relay actuators. In [6,33], as here, results are considering delayed state variables, but they are independent of the delay value and, consequently, may be more conservative than delay-dependent results. In [34], relay is involved in the only identification procedure, then replaced by a finite spectrum assignment control $[24]$.

The present paper considers uncertain systems with single / multiple, constant or time-varying state-delays and additive perturbations which may be nonvanishing. It aims at designing the sliding surface in such a way that the calculable set of admissible delays is maximized where "admissible" means here those that don't destabilize the closed-loop, relay-delay system.

The paper is organized as follows: after some notations, Section 3 is devoted to preliminary results (transformation of the original system into regular form). Then, in Section 4, a sliding mode controller is developed by means of an LMI approach. The reduced system is proven to be asymptotically stable for any value of the delay less than a bound which is obtained by solving a convex optimization problem. This result is generalized to multiple delays. Section 5 presents a sliding mode controller for time-varying delays. Lastly, in Section 6 , two illustrative examples show the effectiveness of the method.

Among the original results, note that taking varying delays into consideration (Section 5) allows us to deal with a large class of uncertainties on the delay value. This is achieved by using a function of Lyapunov-Razumikhin's [17] for the stability study of the reduced system (the system on the surface). This makes the difference with the Lyapunov-Krasovskii's functionals (used in Section 4 for a constant delay $h$ ), since this last approach generally needs additional assumptions on the delay derivative (i.e., $\dot{h}(t) \leq d<1$ ) [27]. Moreover, in Section 5, the implemented controller involves the upperbound of the delay: then, its law of variation has not to be known, which is convenient for 
robustness issues. The same approach could have been followed in Section 4 . But, in order to avoid an unwieldly presentation, we prefered to restrict Section 4 to the case of a known, constant delay, which of course allows for less conservative results.

\section{Notations and assumptions}

In this paper, the following system, with possibly time-varying delay $h=$ $h(t) \geq 0$, will be considered:

$$
\left\{\begin{array}{c}
\dot{x}(t)=A x(t)+A_{d} x(t-h)+B u(t)+f_{1}\left(t, x_{t}\right), t>0 \\
x(t)=\phi(t), \text { for } t \in\left[-h_{\text {sup }}, 0\right], h_{\text {sup }}=\sup _{t \geq 0} h(t), .
\end{array}\right.
$$

The following notations are used: $x(t) \in \mathbb{R}^{n} ; A$ and $A_{d}$ are constant $n \times n$ matrices; $B$ is a $n \times m$ matrix, $u \in \mathbb{R}^{m}$ is the input vector; $f_{1}$ is a term representing the neglected dynamics and external disturbances. The state at time $t$ is the function $x_{t}$ defined by: $x_{t}:[-h, 0] \rightarrow \mathbb{R}^{n}, x_{t}(\theta)=x(t+\theta)$, for $\theta \in[-h, 0] . \phi=x_{t=0}$ is the initial function defined over $\left[-h_{\text {sup }}, 0\right]$, bounded but possibly eventually discontinuous.

$\|e\|$ denotes the Euclidean norm of the $n$-vector $e$, and $\|M\|=\sup _{\|e\|=1}\|M e\|$ is the spectral norm of the $n \times n$ matrix $M$. Finally, $\widetilde{M}$ denotes an orthogonal complement of $M$ (i.e., $\widetilde{M}^{T} M=0$ ).

We will use the following assumptions:

A1) The pair $\left(A+A_{d}, B\right)$ is controllable.

A2) The perturbation term $f_{1}$ satisfies the classical matching conditions, i.e.

$$
f_{1}\left(x_{t}, t\right)=B f\left(x_{t}, t\right)
$$

and is bounded, as follows, by a known functional $\Psi$ of $x_{t}$ :

$$
\|f\|<\Psi\left(x_{t}\right) .
$$

A3) $B$ is full-rank: $\operatorname{rank}(B)=m$.

\section{Regular form}

The aim is to design a sliding mode controller for (2.1). We first transform the original system into a special form, appropriate for sliding mode control 
(classically called regular form [22]). Let us choose the following sliding surface, based on some matrix $X$ to be chosen later (via some optimization):

$$
s(x)=S x=B^{T} X^{-1} x=0,
$$

where $S \in \mathbb{R}^{(m) \times n}$. We also define a nonsingular transformation

$$
z(t)=M x(t), \quad \operatorname{det} M \neq 0,
$$

where

$$
M=\left[\begin{array}{c}
\tilde{B}^{T} \\
S
\end{array}\right] .
$$

Lemma 1 The original system (2.1) is equivalent to

$$
\dot{z}(t)=\hat{A} z(t)+\hat{A}_{d} z(t-h)+\hat{B}(u(t)+f),
$$

where

$$
\begin{aligned}
& \hat{A}=\left(\begin{array}{cc}
\hat{A}_{11} & \hat{A}_{12} \\
\hat{A}_{21} & \hat{A}_{22}
\end{array}\right)=\left(\begin{array}{cc}
\tilde{B}^{T} A X \tilde{B}\left(\tilde{B}^{T} X \tilde{B}\right)^{-1} & \tilde{B}^{T} A B(S B)^{-1} \\
S A X \tilde{B}\left(\tilde{B}^{T} X \tilde{B}\right)^{-1} & S A B(S B)^{-1}
\end{array}\right), \\
& \hat{A}_{d}=\left(\begin{array}{cc}
\hat{A}_{d 11} & \hat{A}_{d 12} \\
\hat{A}_{d 21} & \hat{A}_{d 22}
\end{array}\right)=\left(\begin{array}{cc}
\tilde{B}^{T} A_{d} X \tilde{B}\left(\tilde{B}^{T} X \tilde{B}\right)^{-1} & \tilde{B}^{T} A_{d} B(S B)^{-1} \\
S A_{d} X \tilde{B}\left(\tilde{B}^{T} X \tilde{B}\right)^{-1} & S A_{d} B(S B)^{-1}
\end{array}\right), \\
& \hat{B}=\left(\begin{array}{c}
0 \\
S B
\end{array}\right) \text {. }
\end{aligned}
$$

Proof. The complete proof can be found in [5] as well as $[14,15]$.

In the following, we first present a sliding mode control for a system with one constant delay (Section 4), then the result is extended to the case of systems with several delays and, later on, to a time-varying delay (Section 5).

\section{Sliding mode control synthesis: case of a constant delay}

In this section, we consider that the delay $h$ is constant and known (see the last paragraph of the introduction).

Theorem 1 Let $\Lambda \in \mathbb{R}^{n \times n}$ be a Hurwitz matrix, $P$ the solution of the Lyapunov equation

$$
\Lambda^{T} P+P \Lambda=-I
$$


and $m_{1}>0$ a real number. Assume that conditions $(A 1)-(A 2)-(A 3)$ hold. Then, with the control law

$$
u(t)=-(S B)^{-1}\left(S A x(t)+S A_{d} x(t-h)-\Lambda s+m \frac{P s}{\|P s\|}\right),
$$

where $m=m_{1}+\|S B\| \Psi\left(x_{t}\right)$, system (2.1) exhibits an asymptotically stable sliding mode for any value of the delay $h$ such that $0 \leq h<h_{\max }$, where $h_{\max }$ is the solution of the optimization problem:

$$
h_{\max }^{-1}=\min \left(h^{-1}\right)
$$

subject to the constraints (with $\sigma \in \mathbb{R}$ ):

$$
\begin{aligned}
& \left(\begin{array}{ccc}
h^{-1}\left[X\left(A+A_{d}\right)^{T}+\left(A+A_{d}\right) X\right] & X A_{d}^{T} & X\left(A+A_{d}\right)^{T} \\
A_{d} X & -X & 0 \\
\left(A+A_{d}\right) X & 0 & -X
\end{array}\right) \\
& -\sigma\left(\begin{array}{ccc}
B B^{T} & 0 & 0 \\
0 & B B^{T} & 0 \\
0 & 0 & B B^{T}
\end{array}\right)<0 \\
& X>0 \text {. }
\end{aligned}
$$

Remark 1 In practice, the stable matrix $\Lambda$ allows for choosing the dynamics of $s$ when the solution of the system is far from the manifold $s(x)=0$.

The proof is decomposed into two subproblems: firstly, prove the attractivity of the surface in finite time; secondly, prove the asymptotic stability of the reduced system (on the surface). Note that, since the system has bounded initial functions and is linear during the reaching phase, it cannot diverge during this phase (in finite time). This property would not hold anymore if the system was nonlinear [35].

\subsection{Attractivity of the surface}

Theorem 2 Under assumptions $(A 1)-(A 2)-(A 3)$, the control (4.1) makes the surface $s(x)=0$ stable and globally attractive in finite time.

Proof. Let us consider the function

$$
V(t)=s^{T}(x(t)) P s(x(t))
$$

Its derivative along the trajectories of (3.3) with (4.1) is 


$$
\dot{V}(t)=s^{T}(x)\left(\Lambda^{T} P+P \Lambda\right) s(x)+2 s^{T}(x) P\left(S B f-m \frac{P s}{\|P s\|}\right) .
$$

Then :

$$
\dot{V}(t)=-s^{T}(x) s(x)+2 s^{T}(x) P\left(S B f-m \frac{P s}{\|P s\|}\right) .
$$

As $f$ is bounded, we get :

$$
\dot{V}(t) \leq 2\left(\|S B\| \Psi\left(x_{t}\right)-m\right)\|P s\|,
$$

and then

$$
\dot{V}(t) \leq-2 m_{1}\|P s\| .
$$

We have the following inequality :

$$
\|P s\|^{2}=\left(P^{\frac{1}{2}} s\right)^{T}\left(P^{\frac{1}{2}} s\right) \geq \lambda_{\min }(P)\left\|P^{\frac{1}{2}} s\right\|^{2} .
$$

As $V(t)=s^{T} P s$, then

$$
\left\|P^{\frac{1}{2}} s\right\|^{2}=V(t)
$$

Finally, we get

$$
\dot{V}(t) \leq-2 m_{1} \sqrt{\lambda_{\min }(P)} \sqrt{V(t)} .
$$

This last inequality is known to prove the finite time convergence of the system (3.3) towards the surface [35].

\subsection{Asymptotic stability of the reduced system}

The vector $z$ appearing in the regular form (3.3) is partitioned into $z=\left[z_{1}\right.$ $\left.z_{2}\right]^{T}$, where $z_{1} \in \mathbb{R}^{n-m}, z_{2} \in \mathbb{R}^{m}$.

Once in sliding mode, the equations $s(x)=\dot{s}(x)=0$ lead to the reduced system:

$$
\dot{z}_{1}(t)=\hat{A}_{11} z_{1}(t)+\hat{A}_{d 11} z_{1}(t-h) .
$$

Theorem 3 Under assumptions $(A 1)-(A 2)$, the reduced system (4.7) is asymptotically stable for all delays $h<h_{\max }$, where $h_{\max }$ is solution of the following generalized eigenvalue problem

$$
h_{\max }^{-1}=\min _{X, \sigma}\left(h^{-1}\right)
$$

subject to the constraints (4.3). 
Proof. Consider the Lyapunov-Krasovskii functional

$$
V(t)=y^{T}(t) P y(t)+\int_{t-h}^{t} \int_{s}^{t} z_{1}^{T}(w) \hat{A}_{d 11}^{T} P Q^{-1} P \hat{A}_{d 11} z_{1}(w) \mathrm{d} w \mathrm{~d} s
$$

where $y(t)=z_{1}(t)+\int_{t-h}^{t} \hat{A}_{d 11} z_{1}(w) \mathrm{d} w, P, Q \in \mathbb{R}^{n \times n}$ are two positive-definite matrices. Note that

$$
\begin{aligned}
\dot{y}(t) & =\tilde{B}^{T}\left(A+A_{d}\right) X \tilde{B}\left(\tilde{B}^{T} X \tilde{B}\right)^{-1} z_{1}(t), \\
& =\left(\hat{A}_{d 11}+\hat{A}_{11}\right) z_{1}(t) .
\end{aligned}
$$

Let us denote $Z=\hat{A}_{d 11}+\hat{A}_{11}$. Then, the time-derivative of $V(t)$ is

$$
\begin{aligned}
\dot{V}(t) & =z_{1}(t)\left(Z^{T} P+P Z+h \hat{A}_{d 11}^{T} P Q^{-1} P \hat{A}_{d 11}\right) z_{1}(t) \\
& +2 \int_{t-h}^{t} z_{1}^{T}(w) \hat{A}_{d 11}^{T} P Z z_{1}(t) \mathrm{d} w \\
& -\int_{t-h}^{t} z_{1}^{T}(w) \hat{A}_{d 11}^{T} P Q^{-1} P \hat{A}_{d 11} z_{1}(w) \mathrm{d} w .
\end{aligned}
$$

By using the inequality (see lemma 3 in the appendix)

$$
\begin{aligned}
2 \int_{t-h}^{t} z_{1}^{T}(w) \hat{A}_{d 11}^{T} P Z z_{1}(t) d w & \leq \int_{t-h}^{t} z_{1}^{T}(w) \hat{A}_{d 11}^{T} P Q^{-1} P \hat{A}_{d 11} z_{1}(w) \mathrm{d} w \\
& +h z_{1}(t) Z^{T} Q Z z_{1}(t)
\end{aligned}
$$

the following estimate is derived:

$$
\dot{V}(t) \leq z_{1}(t) M z_{1}(t)
$$

where $M=Z^{T} P+P Z+h\left(\hat{A}_{d 11}^{T} P Q^{-1} P \hat{A}_{d 11}+Z^{T} Q Z\right)$.

We can conclude that, if there exists $h^{*}$ a positive real number and three positive definite matrices $X, P, Q$ such that $M<0$, then the reduced system is asymptotically stable for any value of the delay $h$ such that $0 \leq h \leq h^{*}$.

Nevertheless, choosing the parameters $(P, Q, X)$ so as to optimize the upperbound $h^{*}$ can be a very hard task. In the following, we propose to use a more particular form of (4.9), taking $P=Q=\left(\tilde{B}^{T} X \tilde{B}\right)^{-1}$. By this way, the optimization problem is transformed into a convex one: indeed, by post- and pre-multiplying $M$ by $P^{-1}$ (which is positive definite), the inequality $M<0$ is transformed into the following one:

$$
\begin{gathered}
h^{-1}\left(\tilde{B}^{T} X\left(A+A_{d}\right)^{T} \tilde{B}+\tilde{B}^{T}\left(A+A_{d}\right) X \tilde{B}\right) \\
+\tilde{B}^{T} X\left(A+A_{d}\right)^{T} \tilde{B}\left(\tilde{B}^{T} X \tilde{B}\right)^{-1} \tilde{B}^{T}\left(A+A_{d}\right) X \tilde{B} \\
+\tilde{B}^{T} X A_{d}^{T} \tilde{B}\left(\tilde{B}^{T} X \tilde{B}\right)^{-1} \tilde{B}^{T} A_{d} X \tilde{B}<0 .
\end{gathered}
$$


By using the Schur's complement, the last inequality is shown to be equivalent to

$$
\begin{gathered}
L^{T}\left(\begin{array}{ccc}
h^{-1}\left(H+H^{T}\right) & X A_{d}^{T} & H^{T} \\
A_{d} X & -X & 0 \\
H & 0 & -X
\end{array}\right) L<0, \\
\text { with } L=\left(\begin{array}{ccc}
\tilde{B} & 0 & 0 \\
0 & \tilde{B} & 0 \\
0 & 0 & \tilde{B}
\end{array}\right) \text {, and } H=\left(A+A_{d}\right) X
\end{gathered}
$$

which is also equivalent to (4.3). This last inequality is an LMI in $X$ and $\sigma$ and can be solved very efficiently by convex optimization algorithms.

Remark 2 If we consider the particular case $h=0$, the LMI formulation (4.3) is not convenient anymore but its equivalent equation (4.12) reduces to the simple Lyapunov equation $M=Z^{T} P+P Z<0$ with $Z=\hat{A}_{d 11}+\hat{A}_{11}$. This is a classical result for systems without delay [35].

\subsection{Extension to the multiple delay case}

Consider now the more general system with $k$ constant delays $h_{i}, h=\max _{i}\left(h_{i}\right)$,

$$
\left\{\begin{array}{c}
\dot{x}(t)=A x(t)+\sum_{i=1}^{k} A_{d i} x\left(t-h_{i}\right)+B u(t)+f_{1}, \text { for } t>0, \\
x(t)=\phi(t), \text { for } t \in[-h, 0]
\end{array}\right.
$$

where $A_{d i}$ are $k$ constant $n \times n$ matrices, the other notations being unchanged. The assumptions $(A 1)$ and $(A 2)$ will be replaced by:

$\left(A^{\prime} 1\right)\left(A+\sum_{i=1}^{k} A_{d}, B\right)$ is controllable.

$\left(A^{\prime} 2\right)\|f\|<\Psi\left(x_{t}\right)$.

Theorem 4 Let $\Lambda \in \mathbb{R}^{n \times n}$ be a Hurwitz matrix, $P$ the solution of the Lyapunov equation

$$
\Lambda^{T} P+P \Lambda=-I,
$$

and $m_{1}>0$ a real number. Assume that conditions $\left(A^{\prime} 1\right)-\left(A^{\prime} 2\right)-(A 3)$ hold. Then, with the control law

$$
u(t)=-(S B)^{-1}\left(-\Lambda s(x)+S A x(t)+\sum_{i=1}^{k} S A_{d i} x\left(t-h_{i}\right)+m \frac{P s}{\|P s\|}\right)
$$


where $m=m_{1}+\|S B\| \Psi\left(x_{t}\right)$, system (4.15) exhibits an asymptotically stable sliding mode for any values of the delays $h_{i}$ such that $0 \leq h_{i}<h_{i \max }(i=$ $1, \ldots, k)$, where the parameters $h_{i \max }$ satisfy the following LMIs:

$$
\begin{aligned}
& \left(\begin{array}{ccccc}
J^{T}+J & h_{1 \max } X A_{d 1}^{T} & \ldots & h_{k \max } X A_{d k}^{T} & \sum_{i=0}^{k} h_{\text {imax }} J^{T} \\
h_{1 \max } A_{d} X & -h_{1 \max } X & 0 & 0 & 0 \\
\vdots & 0 & \ddots & 0 & 0 \\
h_{k \max } A_{d k} X & 0 & 0 & -h_{k \max } X & 0 \\
\sum_{i=0}^{k} h_{i \max } J & 0 & 0 & 0 & -\sum_{i=0}^{k} h_{\text {imax }} X
\end{array}\right) \\
& -\left(\begin{array}{ccccc}
B B^{T} & 0 & 0 & 0 & 0 \\
0 & B B^{T} & 0 & 0 & 0 \\
0 & 0 & \ddots & 0 & 0 \\
0 & 0 & 0 & B B^{T} & 0 \\
0 & 0 & 0 & 0 & B B^{T}
\end{array}\right)<0 \\
& X>0 \\
& -\sigma
\end{aligned}
$$

where $J=\left(A+\sum_{i=0}^{k} A_{d i}\right) X, \sigma \in \mathbb{R}$.

Proof. The proof is similar to the proof of Theorem 1.

\section{Sliding mode control synthesis: case of a time-varying delay.}

In this section, we consider that the delay $h=h(t)$ is varying. Its variation law can be unknown, but it is supposed to remain uniformly bounded by some constant $h_{\max }$. The system is then defined by

$$
\left\{\begin{array}{c}
\dot{x}(t)=A x(t)+A_{d} x(t-h(t))+B u(t)+f_{1}, t>0, \\
x(t)=\phi(t), \text { for } t \in\left[-h_{\max }, 0\right] \\
0 \leq h(t) \leq h_{\max } \text { for } t \in\left[-h_{\max },+\infty[\right.
\end{array}\right.
$$

Theorem 5 Let $\Lambda$ be a Hurwitz matrix, $P$ the solution of the Lyapunov equation $\Lambda^{T} P+P \Lambda=-I$, and $m_{1}>0$ a real number. Assume that conditions $(A 1)-(A 3)$ hold. Then, with the control law 


$$
\begin{aligned}
u(t)= & -(S B)^{-1}\left(S A x(t)-\Lambda s+m \frac{P s}{\|P s\|}\right), \\
m= & m_{1}+\|S B\| \sup _{s \in\left[-h_{\max }, 0\right]}\left(\Psi\left(x_{t}(s)\right)\right) \\
& +\left\|S A_{d}\right\| \sup _{s \in\left[-h_{\max }, 0\right]}(\|x(t+s)\|),
\end{aligned}
$$

system (5.1) exhibits an asymptotically stable sliding mode for any delay $h(t)$ such that $h(t) \in\left[0, h_{\max }\right]$, where $h_{\max }$ is the solution of the following optimization problem

$$
h_{\max }^{-1}=\min \left(h^{-1}\right)
$$

with the LMI-constraints

$$
\begin{aligned}
& \left(\begin{array}{cc}
\alpha X & A X \\
X A^{T} & X
\end{array}\right)-\gamma_{1}\left(\begin{array}{cc}
B B^{T} & 0 \\
0 & B B^{T}
\end{array}\right)>0 \\
& \left(\begin{array}{cc}
\alpha X & A_{d} X \\
X A_{d}^{T} & X
\end{array}\right)-\gamma_{2}\left(\begin{array}{cc}
B B^{T} & 0 \\
0 & B B^{T}
\end{array}\right)>0 \\
& \left(\begin{array}{cc}
h^{-1}\left(H^{T}+H+(\alpha+\beta) X\right. & A_{d} X \\
X A_{d}^{T} & -\frac{1}{2} X
\end{array}\right)-\sigma\left(\begin{array}{cc}
B B^{T} & 0 \\
0 & B B^{T}
\end{array}\right)<0, \\
& X>0,
\end{aligned}
$$

where $H=\left(A+A_{d}\right) X, \gamma_{1}, \gamma_{2}, \sigma \in \mathbb{R}, \alpha, \beta \in \mathbb{R}^{+}$.

Note that if $\alpha, \beta \in \mathbb{R}^{+}$is fixed, then we have to deal with a generalized eigenvalue problem (GEVP). Then, a relaxation-type theorem to $\alpha, \beta$ can be used so to to improve the upperbound of $h_{\max }$.

Proof. As previously, the proof is achieved in two parts: attractivity of the surface $s(x)=0$ and stability of the reduced system. The Proof of the first part can be done as previously, so we won't repeat it here. The proof of the second part is the following:

Once in sliding mode, the equations $s(x)=\dot{s}(x)=0$ lead to the reduced system:

$$
\dot{z}_{1}(t)=\hat{A}_{11} z_{1}(t)+\hat{A}_{d 11} z_{1}(t-h) .
$$

By using the Leibnitz-Newton formula, we have:

$$
\dot{z}_{1}(t)=\left(\hat{A}_{11}+\hat{A}_{d 11}\right) z_{1}(t)-\int_{t-h}^{t} \hat{A}_{d 11} \dot{z}_{1}(v) d v
$$


So, the reduced system can be rewritten in the form

$$
\begin{aligned}
\dot{z}_{1}(t) & =\left(\hat{A}_{11}+\hat{A}_{d 11}\right) z_{1}(t)-\int_{t-h}^{t} \hat{A}_{d 11} \hat{A}_{11} z_{1}(v) d v \\
& -\int_{t-h}^{t} \hat{A}_{d 11} \hat{A}_{d 11} z_{1}(v-h) d v .
\end{aligned}
$$

Consider the following function

$$
V(t)=z_{1}^{T}(t) P z_{1}(t)
$$

where $P$ is a symmetric, positive-definite matrix of appropriate dimension. Following Razumikhin's approach, we assume that the inequality

$$
V(x(t+\theta))<q V(x(t))
$$

with $q>1$ holds for any $\theta \in\left[-h_{\max }, 0\right]$. The time-derivative of $V$ is

$$
\begin{aligned}
\dot{V} & =z_{1}^{T}(t)\left(Z^{T} P+P Z\right) z_{1}(t)-2 \int_{t-h}^{t} z_{1}^{T}(t) P \hat{A}_{d 11} \hat{A}_{11} z_{1}(v) d v \\
& -2 \int_{t-h}^{t} z_{1}^{T}(t) P \hat{A}_{d 11}^{2} z_{1}(v-h) d v
\end{aligned}
$$

where, again, $Z=\hat{A}_{11}+\hat{A}_{d 11}$.

Using Lemma 3 and inequality (5.12), we derive the following upperbounding of $\dot{V}(t)$ :

$$
\dot{V}(t)<z_{1}^{T}(t) N z_{1}(t),
$$

where $N=Z^{T} P+P Z+h \alpha^{-1} P \hat{A}_{d 11} \hat{A}_{11} S \hat{A}_{11}^{T} \hat{A}_{d 11}^{T} P+q h(\alpha+\beta) P+h \beta^{-1} P \hat{A}_{d 11}^{2} S \hat{A}_{d 11}^{2 T} P$.

We can conclude that if there exist $h^{*}$ and $q$ such that $N$ is negative-definite, then the reduced system is uniformly asymptotically stable.

By post- and pre-multiplying by $S$, and fixing $S=\left(\tilde{B}^{T} X \tilde{B}\right), N<0$ becomes:

$$
S Z^{T}+Z S+h \alpha^{-1} \hat{A}_{d 11} \hat{A}_{11} S \hat{A}_{11}^{T} \hat{A}_{d 11}^{T}+q h(\alpha+\beta) S+h \beta^{-1} \hat{A}_{d 11}^{2} S \hat{A}_{d 11}^{2 T}<0 .
$$

Let us assume that (5.4) and (5.5) hold. Using Lemma 2, these inequalities can be reformulated as, respectively,

$$
\tilde{B}^{T} A X \tilde{B}\left(\tilde{B}^{T} X \tilde{B}\right)^{-1} \tilde{B}^{T} X A^{T} \tilde{B}<\alpha\left(\tilde{B}^{T} X \tilde{B}\right)
$$

and

$$
\tilde{B}^{T} A_{d} X \tilde{B}\left(\tilde{B}^{T} X \tilde{B}\right)^{-1} \tilde{B}^{T} X A_{d}^{T} \tilde{B}<\beta\left(\tilde{B}^{T} X \tilde{B}\right)
$$


From these two inequalities and (5.14), we prove that

$$
\begin{gathered}
\left(\tilde{B}^{T} X\left(A+A_{d}\right)^{T} \tilde{B}+\tilde{B}^{T}\left(A+A_{d}\right) X \tilde{B}\right)+q h(\alpha+\beta)\left(\tilde{B}^{T} X \tilde{B}\right) \\
+h \tilde{B}^{T} A_{d} X \tilde{B}\left(\tilde{B}^{T} X \tilde{B}\right)^{-1} \tilde{B}^{T} X A_{d}^{T} \tilde{B}+h \tilde{B}^{T} A_{d} X \tilde{B}\left(\tilde{B}^{T} X \tilde{B}\right)^{-1} \tilde{B}^{T} X A_{d}^{T} \tilde{B}<0,
\end{gathered}
$$

which, by virtue of Lemma 3, is equivalent to

$$
\left(\begin{array}{cc}
h^{-1}\left(H^{T}+H+q(\alpha+\beta) X\right. & A_{d} X \\
X A_{d}^{T} & -\frac{1}{2} X
\end{array}\right)-\sigma\left(\begin{array}{cc}
B B^{T} & 0 \\
0 & B B^{T}
\end{array}\right)<0 .
$$

For $q=1$, this last inequality is exactly the constraint. So, by continuity, if we assume that there are parameters $X, h, \sigma, \alpha, \beta$ such that (29) holds, then there exists $q_{1}>1$ such that (5.16) holds also. This completes the proof.

Remark 3 As in the previous Remark 2, the particular case $h=0$ reduces (5.14) to a classical Lyapunov equation concerning the stability of the reduced system (on the surface) [4].

\section{Examples}

\subsection{Example 1}

Consider the following example:

$$
\begin{aligned}
& \dot{z}(t)=A z(t)+A_{d} z(t-h)+B(u(t)+f(t)) \\
& A=\left(\begin{array}{ccc}
2 & 0 & 1 \\
1.75 & 0.25 & 0.8 \\
-1 & 0 & 1
\end{array}\right) \\
& A_{d}=\left(\begin{array}{ccc}
-1 & 0 & 0 \\
-0.1 & 0.25 & 0.2 \\
-0.2 & 4 & 5
\end{array}\right), B=\left(\begin{array}{l}
0 \\
0 \\
1
\end{array}\right) \\
&\|f(t)\| \leq 2, f(t)=2 \sin (t) \text { for simulation purpose, } \\
& z(t)=1, t \in[-h, 0] .
\end{aligned}
$$

Constant delay: By using semi-definite programming, we find that the system (6.1) with control (4.1) is asymptotically stable for all constant delays $h<$ 0.99. The simulation provided in Figure 1 was obtained with a first-order integration scheme of step 0.01 . 
Varying delay: Now, if we don't restrict to constant delays, then according to Theorem 5, the system is asymptotically stabilized by the control law (5.1) for $h(t)<0.51$ which is of course more constraining. The simulation leads to Figures 2 and 3.

\subsection{Example 2}

Consider system (2.1) with a constant delay, which stabilization has been considered in $[13,16,21]$ :

$$
A=\left[\begin{array}{cc}
2 & 0 \\
1.75 & 0.25
\end{array}\right], A_{d}=\left[\begin{array}{cc}
-1 & 0 \\
-0.1 & -0.25
\end{array}\right], B=\left[\begin{array}{l}
1 \\
1
\end{array}\right]
$$

As the pair $\left(A, A_{d}\right)$ is not controllable, the system cannot be stabilized independently of the delay. By applying Theorem 1, we find the results summarized in Table 1.

Table 1

Results for Example 2

\begin{tabular}{|l|l|l|}
\hline & delay upperbound & parameters \\
\hline Theorem 1 & 1.65 & $s(x)=4.4610^{-4} x_{1}(t)+1.39210^{-4} x_{2}(t)$ \\
\hline Ivanescu et al.[16] & 1.46 & \\
\hline Fu et al.[13] & 0.984 & \\
\hline Li and De Souza[21] & 0.51 & \\
\hline
\end{tabular}

\section{Conclusion}

The contribution of this paper lays on the following, original points:

- Concerning the design of linear sliding surface, the extension of the method [6] to the case of a delay-dependent stabilization (then, conditions are less conservative). The gain of the surface is expressed as a solution of an LMI problem which allows for easy computations.

- The consideration of multiple, constant delays.

- The consideration of a time-varying delay. This point is probably the most important for robustness issues (with regard to bad delay identification). The 
proposed controller (30) does not refer to the variation law of the delay $h(t)$, but to its maximum value.

- The results of Section 4 can be extended to unknown constant delay, as well as those of Section 5 to multiple delay: however, the presentation would then turn to be cumbersome.

Lastly note that the sliding mode strategy is straightforward, easy to implement and of reduced complexity since the stabilization problem has been reduced to two subproblems of lower dimensions. This explains why the result obtained on Example 2 improves previous ones.

\section{Appendix}

The following result.is used in the proofs of Theorems $3,4,5$.

Lemma 2 (see [4] p. 33) The inequality

$$
\tilde{U}^{T} G \tilde{U}>0
$$

is equivalent, for some $\sigma \in \mathbb{R}$, to

$$
G-\sigma U^{T} U>0 .
$$

Lemma 3 For two vectors $x, y$ of $\mathbb{R}^{n}$ and a positive-definite matrix $X \in \mathbb{R}^{n \times n}$, the following inequality holds:

$$
x^{T} y \leq x^{T} X^{-1} x+y^{T} X y
$$



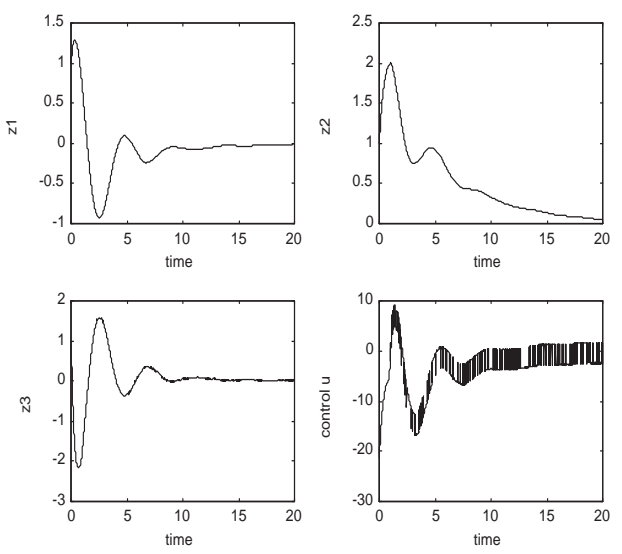

Fig. 1. Response of (6.1) with control (4.1) and $h=0.9$.
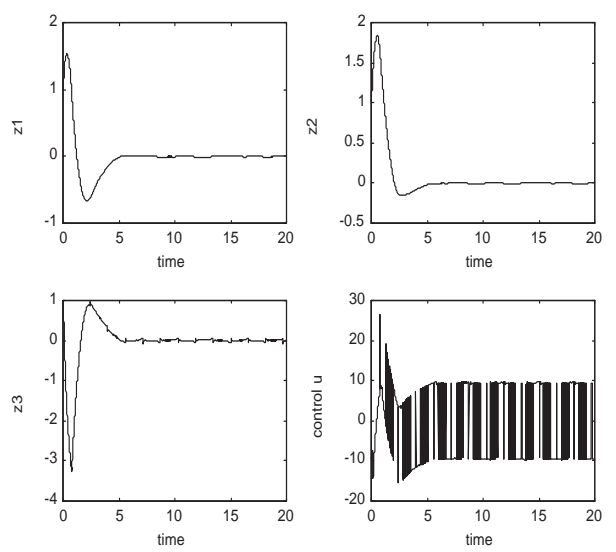

Fig. 2. Response of (6.1) with control (4.1) and $h(t)<h_{\max }=0.51$.

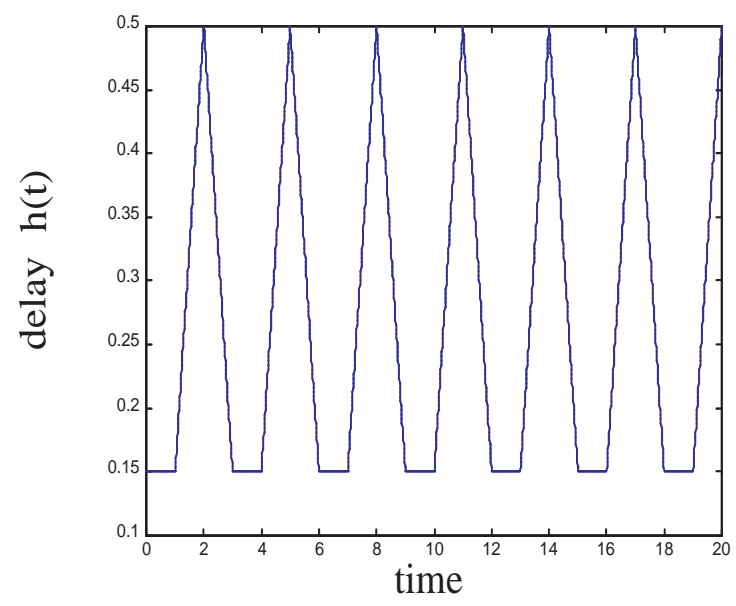

Fig. 3. Implemented time-varying delay

\section{References}

[1] W. Aggoune. Contribution à la Stabilisation de Systèmes Non Linéaires: Application Aux Systèmes Non Réguliers et Aux Systèmes à Retards. PhD 
thesis, INRIA Lorraine/CRAN, Univ. de Metz, France, 1999.

[2] C. Bonnet, J.R. Partington, and M. Sorine. Robust control and tracking in $1_{\infty}$ of delay systems equipped with a relay sensor. In Proc. 8th. IFAC Conf. Large Scale Systems LSS'98, Patras, 1998.

[3] C. Bonnet, J.R. Partington, and M. Sorine. Robust stabilization of a delay system with saturating actuator or sensor. International Journal of Robust and Nonlinear Control, 10(7) (2000) 579-590.

[4] S. Boyd, El-Ghaoui L., E. Feron, and V. Balakrishan. Linear Matrix Inequalities in System and Control Theory. SIAM, Philadelphia, (1994).

[5] H.H. Choi. A new method for variable structure control system design : A linear matrix inequality approach. Automatica, 33 (1997) 2089-2092.

[6] H.H. Choi. An LMI approach to sliding mode control design for a class of uncertain time delay systems. In Proc. ECC'99, Karlsruhe, Germany, 1999.

[7] G. Conte and A.M. Perdon. The disturbance decoupling problem for systems over a ring. SIAM J. Control and Optimization, 33(3)(1995).

[8] G. Conte and A.M. Perdon. Systems over rings: Theory and applications. In Proc. IFAC Workshop LTDS'98, Linear Time Delay Systems, pages 223-234, Grenoble, France, 1998. Plenary lecture.

[9] M. Dambrine, F. Gouaisbaut, W. Perruquetti, and J.-P. Richard. Robustness of sliding mode control under delays effects: a case study. In Proc. 2nd IEEE-IMACS CESA'98, Comput. Eng. In Systems Applications, pages 817821, Hamamet, Tunisie, 1998.

[10] M. Dambrine, J.P. Richard, and P. Borne. Feedback control of timedelay systems with bounded control and state. Mathematical Problems in Engineering, (1) (1995) 77-87.

[11] L. Dugard and E.I. Verriest, editors. Stability and Control of Time-Delay Systems, volume 228 of LNCIS. Springer Verlag, (1997).

[12] L.M. Fridman, E. Fridman, and E.I. Shustin. Steady modes and sliding modes in the relay control systems with time delay. In 35th IEEE CDC'96, pages 4601-4606, Kobe, Japan, 1996.

[13] M. Fu, H. Li, and S.I. Niculescu. Robust Stability and Stabilisation of TimeDelay System Via Integral Quadratic Constraint Approach, volume 228 of LNCIS, pages 101-116. Springer-verlag edition, 1997.

[14] F. Gouaisbaut, W. Perruquetti, Y. Orlov, and J.P. Richard. A sliding mode controller for linear time delay systems. In Proc. ECC'99 European Control Conference, Karlsruhe, September 1999.

[15] F. Gouaisbaut, W. Perruquetti, and J.P. Richard. A sliding mode control for linear systems with input and state delays. In Proc. 38th IEEE CDC'g9, Phoenix, 1999. 
[16] D. Ivanescu. Sur la Stabilisation Des Systèmes À Retard : Théorie et Applications. PhD thesis, Institut National Polytechnique de Grenoble, 2000.

[17] V.B. Kolmanovskii and A. Myshkis. Introduction to the theory and applications of functional differential equations. Kluwer Acad., Dordrecht, (1999).

[18] V.B. Kolmanovskii, S.I. Niculescu, and J.P. Richard. On the LiapunovKrasovskii functionals for stability analysis of linear delay systems. Int. J. Control, 72(4) (1999) 374-384.

[19] A.J. Koshkouei and A.S.I. Zinober. Sliding mode time-delay systems. In Proc. International Workshop on VSS, pages 97-101, Tokyo, 1996.

[20] X. Li and C.E. De Souza. Robust stabilization and $\mathrm{H}_{\infty}$ control of uncertain linear time-delay systems. In Proc. 13th IFAC World Congress, pages 113-118, San Francisco, CA, 1996. Vol. H.

[21] X. Li and C.E. De Souza. Delay-dependent robust stability and stabilization of uncertain linear delay systems : A linear matrix inequality approach. IEEE Trans. Aut. Control, 42(8) (1997) 1144-1148.

[22] A.G. Lukyanov and V.I. Utkin. Methods of reducing equations of dynamics systems to regular form. Automat. Remote Control, 42 (1981) 413-420.

[23] N. Luo and M. De la Sen. State feedback sliding mode control of a class of timedelay systems. In ACC'92 (American Control Conf.), pages 894-895, Chicago, Illinois, USA, 1992.

[24] A. Manitius and A.W. Olbrot. Finite spectrum assignment problem for systems with delays. IEEE Trans. Aut. Control, 24(4) (1979) 541-553.

[25] S.K. Nguang. Robust $\mathrm{H}_{\infty}$ control of a class of nonlinear systems with delayed state and control: A LMI approach. In Proc. 37th IEEE CDC'98, pages 23842389, Tampa, Florida, USA, 1998.

[26] S.I. Niculescu. $\mathrm{H}_{\infty}$ memoryless control with an $\alpha$-stability constraint for time delays systems: an LMI approach. IEEE Trans. Aut. Control, 43(5) (1998) 739-743.

[27] S.I. Niculescu, C.E. De Souza, L. Dugard, and J.M. Dion. Robust exponential stability of uncertain systems with time-varying delays. IEEE Trans. Aut. Control, 43(5) (1998) 743-748.

[28] A.W. Olbrot. A sufficiently large time delay in feedback loop must destroy exponential stability of any decay rate. IEEE Trans. Aut. Control, 29 (1984) $367-368$.

[29] P. Picard, J.F. Lafay, and V. Kucera. Model matching for linear systems with delays and 2-d systems. Automatica,, 34(2)(1998).

[30] J.P. Richard. Some trends and tools for the study of time delay systems. In Proc. 2nd IMACS-IEEE CESA'98, pages 27-43, Tunisia, 1998. plenary lecture. 
[31] J.P. Richard, F. Gouaisbaut, and W. Perruquetti. Sliding mode control in the presence of delay. Kibernética., 37 (2001) 277-294.

[32] Y.H. Roh and J.H. Oh. Robust stabilization of uncertain input-delay systems by sliding mode control with delay compensation. Automatica, 35 (1999) 16811685 .

[33] K.K. Shyu and J.J. Yan. Robust stability of uncertain time-delay systems and its stabilization by variable structure control. Int. J. Control, 57 (1993) 237-246.

[34] K.K. Tan, Q.K. Wang, and T.H. Lee. Finite spectrum assignment control of unstable time delay processes with relay tuning. Ind. Eng. Chem. Res., 37(4) (1998) 1351-1357.

[35] V.I. Utkin. Sliding modes in control optimization. CCES. Springer-Verlag, (1992). 